\title{
Aggressive incidents on a psychiatric intensive care unit
}

original

papers

\section{AIMS AND METHOD}

This study looked at patient aggressive behaviour on an Irish psychiatric intensive care unit, and whether it was related to diagnosis, patient's insight and symptomatology. Each aggressive incident was recorded throughout the patient's stay using the Staff-Observed Aggression Scale.

\author{
RESULTS \\ Ninety-nine individuals were \\ admitted to the unit during the study. \\ We recorded 82 aggressive incidents, \\ with most occurring during the \\ daytime and on weekdays. There was \\ no statistical difference in BPRS \\ scores between the aggressive and \\ non-aggressive groups. The aggres- \\ sive patient group had a lower insight \\ score than the non-aggressive
}

\begin{abstract}
group $(P<0.05)$ as measured on the Schedule of the Assessment of Insight. However, when gender and verbal aggression only were included in the analysis, the difference in insight was less significant $(P=0.07)$.

\section{CLINICAL IMPLICATIONS}

Aggression is common on a psychiatric intensive care unit. Low levels of insight in patients may increase the risk of aggression.
\end{abstract}

Violence and aggression are major factors affecting morale of psychiatric in- and out-patients and staff, with most of the violent acts being perpetrated by individuals with schizophrenia (Rossi et al, 1985; Tardiff et al, 1997). Established risk factors for assault in this subgroup include a history of violence (Tardiff et al, 2007) and comorbid substance misuse (Swanson et al, 1990). More recent studies suggest a need for special education (Walsh et al, 2004) and involuntary admission (Foley et al, 2005) can also contribute. Staff characteristics (age, length of work experience, training programme in assault prevention and management, frequent changes in staff and use of temporary staff) can also be a factor in patients' assaultive behaviour (James et al, 1990; Chou et al, 2002). An Italian audit showed that approximately half of all aggressive incidents on a psychiatric intensive care unit had no specific cause, occurred during daytime and in the first week of admission and, in most cases, were directed towards staff, other in-patients and visitors (Grassi et al, 2001).

Individuals with schizophrenia who commit violent acts have deficits of insight, including insight into their illness and awareness of the legal implications of their behaviour (Buckley et al, 2004). The relationship between lack of insight and illness in such individuals is important, but under-researched.

Psychiatric intensive care is for in-patients compulsorily detained, usually in secure units, who are in an acutely disturbed phase of a serious mental disorder. The loss of capacity for self-control, which increases risk, does not allow for safe treatment on an open general ward. Length of stay on an intensive care ward would not ordinarily exceed 8 weeks (Department of Health, 2002). Although factors related to in-patient aggression have been extensively studied, there is little specific information about violence in psychiatric intensive care units (Saverimuttu et al, 2000). Research on the prevalence and causes of in-patient aggression in general has been hindered by the use of different methods for measuring aggression (Nijman et al, 1997). Many studies have focused on specific groups of in-patients, such as forensic or first episode psychosis subgroups. Insight assessment of violent in-patients so far has also been completed largely on forensic in-patients only. We prospectively assessed the incidence, insight and clinical correlates of all individuals, irrespective of diagnosis, admitted to a psychiatric intensive care unit over a 10week period.

\section{Method}

The study was carried out on the 18-bed psychiatric intensive care unit at St John of God Hospital, Stillorgan, Dublin county. The hospital is an in-patient psychiatric facility which accepts admissions from all over Ireland, but in-patients mainly come from a predominantly urban, geographically defined catchment area of approximately 165000 people. All individuals admitted or transferred to the psychiatric intensive care unit from other wards were included in the study. Once the person was discharged or transferred to a different ward after their stay in the unit, they were no longer considered to be part of the study. Ninety-nine patients were admitted to the unit during this time. Average bed occupancy for the duration of the study was $95 \%$. Six nurses staff the unit during the day and five nurses at night, with a male/female ratio of 2:3. However, one-to-one special nursing was occasionally employed in the unit for patients requiring a higher level of observation and care.

On admission to the unit, the Brief Psychiatric Rating Scale (BPRS; Overall et al, 1962) and Schedule for the Assessment of Insight (SAl; David, 1990) were completed on each individual by the doctor on duty, and biographical data such as age, gender, marital status and previous admissions were recorded. The five BPRS subscales (hostile/suspiciousness, anxious/depressed, withdrawn/ retarded, agitated/excited, thinking disturbance) were also used in the analysis. The SAl examines insight for illness, treatment and psychotic phenomena. As many individuals were not psychotic, insight scores were only examined for illness and treatment. 
Each verbal and non-verbal aggressive incident was recorded throughout the person's stay by nursing staff using the Staff-Observed Aggression Scale Revised original papers
(Nijman et al, 1999, 2005). The scale measures verbal and physical aggression, including the target of the aggression, the consequences of the act on the target and measures taken to stop the aggression. Before the study commenced, all staff were trained in the use of these instruments and achieved appropriate levels of interrater reliability (Cronbach's $\alpha=0.69$ ). All diagnoses were recorded using DSM-IV criteria by examination of case notes.

The study was approved by the St John of God Provincial Ethics Committee. Statistical analysis was performed using SPSS version 15.0 for Windows.

\section{Results}

\section{Descriptive statistics}

There is no significant difference in mean age by gender of all individuals admitted to the unit during the study (men, 39 years $v$. women, 41 years), but there were significantly more females than males in the group of the $50-59$ year olds (Fig. 1). Regarding patient demographics, $65 \%$ were single, $11 \%$ separated or divorced and $24 \%$ married. Almost two out of five patients (41\%) were unemployed, with $39 \%$ in full-time employment, $11 \%$ in full-time education and $9 \%$ working in the home. Various diagnostic categories for this patient group are shown in Table 1, along with the number and percentage of inpatients in each category who behaved aggressively.

\section{Aggressive incidents}

Eighty-two aggressive incidents were recorded during the study period, including incidents against 26 patients. The majority of patients involved in the incidents were involved in only one $(n=10)$ or two $(n=8)$. Most incidents happened between Monday and Thursday (75\%), with just 25\% between Friday and Sunday. Only $7 \%$ of incidents happened between 11pm and 7am. Men and women were involved in a similar number of aggressive incidents ( $26 \%$ v. $27 \%$ respectively), although men were more likely to use physical aggression ( $22 \%$ v. $14 \%$ respectively; Table 2).

Triggers for the aggression were often unclear, with no understandable provocation in $63 \%$ of aggressive incidents. Staff's denial of patient's requests was the next most common trigger at $21 \%$, and $11 \%$ of incidents happened because the person was provoked by fellow patient(s). In $4 \%$ of incidents, the reason was staff requesting the patient to take medication.

Verbal aggression occurred in $83 \%$ of incidents and physical aggression (e.g. kicking, hitting, throwing objects) in $45 \%$ of incidents. Aggression (whether verbal or physical) was mostly directed against ward staff (63\%) and other patients (36\%). Auto-aggression was present in just $2 \%$ of incidents. Oral or intramuscular medication was required in $21 \%$ of incidents. In $26 \%$ of cases, patients were removed from the general ward area, with

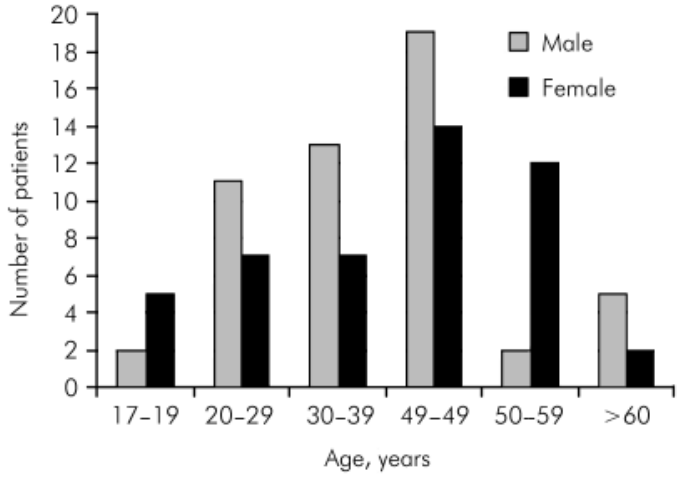

Fig. 1. Age and gender profile of patients.

Table 1. Diagnostic categories

\begin{tabular}{lcc}
\hline Diagnostic group & $n$ & $\begin{array}{c}\text { Aggressive } \\
\text { behaviour } \\
n(\%)\end{array}$ \\
\hline Bipolar affective disorder & 23 & $8(35)$ \\
Schizophrenia & 26 & $6(23)$ \\
Depression & 16 & $1(6)$ \\
Personality disorder & 9 & $2(22)$ \\
Substance misuse & 8 & $1(13)$ \\
Schizoaffective disorder & 7 & $5(71)$ \\
Other psychoses & 6 & $1(17)$ \\
Others & 4 & $2(50)$ \\
Total & 99 & $26(26)$ \\
Gender & & $13(26)$ \\
$\quad$ Male & 50 & $13(27)$ \\
$\quad$ Female & 49 & \\
\hline
\end{tabular}

Table 2. Group and gender

\begin{tabular}{lcc} 
Group & Male, ${ }^{1} n(\%)$ & Female, $^{2} n(\%)$ \\
\hline Non-aggressive & $37(74)$ & $36(74)$ \\
Verbal aggression only & $2(4)$ & $6(12)$ \\
Physical and verbal aggression & $11(22)$ & $7(14)$ \\
\hline 1. $n=50$. & & \\
2. $n=49$.
\end{tabular}

$13 \%$ placed in a time-out area (unlocked room) and $16 \%$ placed in seclusion (locked room). Only $6 \%$ of incidents resulted in a patient being physically restrained.

\section{Clinical correlates of aggression with symptomatology and insight}

For the purpose of analysis, patients were divided by gender into aggressive and non-aggressive groups. During their stay in the psychiatric intensive care unit, $26 \%$ of patients were aggressive and $74 \%$ were not. Initial statistical analysis revealed a significant difference between SAI levels of insight for illness and treatment in the two groups (maximum score 10 for no insight, 0 for full insight), with lower insight scores in the aggressive group (4.34 v. 6.11, $P<0.05$ ). Mean BPRS scores between 
these two groups ( 46.7 v. $43.8, P=0.35$ ) were not statistically significant.

Recent research shows that although verbal aggression is common, it is not indicative of levels of physical aggression (Foley et al, 2005). Taking this into consideration, the aggressive group was further divided into two groups who displayed either verbal aggression only or physical aggression (Table 2). Using univariate analysis of variance (ANOVA), P-values were calculated by group and gender for BPRS total and subscale scores, and SAI scores. The significant difference between aggressive and non-aggressive groups' insight scores was diminished when gender difference and the verbally aggressive group were accounted for in the analysis. However, there was lower insight in the physically aggressive group $(P=0.07)$.

Differences between gender and group for the BPRS total scores were not statistically significant, but significant results were recorded in the anxious/depression subscales $(P<0.05)$ with a higher mean score for the non-aggressive group. The non-aggressive group had a much lower mean score than the aggressive group on the hostile/suspiciousness subscale $(P<0.05)$. Men in the aggressive group had higher mean scores than women on the hostile/suspiciousness and agitation/excitement subscales ( $P<0.05$ for both subscales).

\section{Discussion}

This study provides the first prospective review of in-patients in an Irish psychiatric intensive care unit. Patients on the unit were typically aged 40 , likely to be single $(65 \%)$ and unemployed $(40 \%)$, with an even gender split. As in other studies on psychiatric in-patients' aggressive behaviour (Tardiff et al, 1997), most aggressive patients had a diagnosis of either schizophrenia or bipolar disorder.

Our study shows, in line with other studies (Shah et al, 1991; Nijman et al, 1997), that aggression is common on a psychiatric intensive care unit and that the target is likely to be a member of staff (63\%) or a fellow patient (36\%). Many incidents involved multiple targets. Men were more likely than women to engage in physically aggressive behaviour. Few incidents resulted in injury with just $2 \%$ requiring minor treatment. This is consistent with other studies (Steinert et al, 1999; Foley et al, 2005). Aggressive incidents were more likely to occur on weekdays and during the day, possibly because the ward was busier at that time, resulting in a more disruptive environment for potentially volatile patients.

Physical aggression appeared to be less common than in other studies (45\% in our study v. $60 \%$ of incidents reported in Shah et al, 1991; Nijman et al, 1997; El-Badri \& Mellsop, 2006). Staff reported no clear understandable provocation in $63 \%$ of aggressive incidents, a much higher number than in previous studies (Nijman et al, 1997). Otherwise, staff denial of patient requests was the next most common trigger at $21 \%$, with $11 \%$ of incidents provoked by fellow patients. This is more consistent with previous studies (Nijman et al, 1997).
Admission criteria to a psychiatric intensive care unit commonly include:

- behavioural difficulties which seriously compromise physical or psychological well-being of the patient or others and which cannot be safely assessed or treated in an open acute in-patient facility

- significant risk of aggression or absconding

- risk of suicide or vulnerability (e.g. due to sexual disinhibition or overactivity) in a serious mental disorder (Department of Health, 2002).

Such criteria many account for patients unlikely to display aggression admitted to a psychiatric intensive care unit. Such low-risk patients may be exposed to assaults by a minority of aggressive patients. This study points clearly to that risk, given that $74 \%$ of patients did not display aggressive behaviour, yet the target of aggression in $36 \%$ of incidents were other patients.

Other studies have reported that most patients exhibiting aggressive behaviour were secluded at some point and that physical restraint was required for a third of individuals (El-Badri \& Mellsop, 2006). In this study, $46 \%$ of patients were secluded and only $6 \%$ required physical restraint.

Literature on insight deficits remains sparse (Buckley et al, 2004). Previous studies have focused exclusively on individuals with schizophrenia. This is the first study which has looked at insight in a general group of patients while looking at aggression and showed acceptable levels of interrater reliability (Cronbach's $\alpha=0.69$ ). Insight deficits prevailed in the physically aggressive group, which suggests that they may be predictive of aggression. Further work may reveal insight deficits in aggressive patients in larger sample sizes of diagnostic groups.

The study provides an easy-to-repeat format and promotes multidisciplinary involvement of nursing and medical staff in research. It is possible that aggressive incidents were under-reported by staff. There are many reasons for this, including acceptance as part of routine work, gender of the staff involved and bureaucratic structures that lead to under-reporting (Ferns, 2006). As this was a prospective study from the time of admission, it did not include pre-admission aggression, which may have led to underestimating aggression further.

Given that insight levels were lower in the aggressive group, improved communication with patients about their care and treatment should take place from the outset in formal care planning of patients with lower levels of insight. Aggression remains common in therapeutic environments and staff are commonly the target, as shown by this and other studies. Recent changes in workplace legislation in the UK, for example, have put the onus on employers and management to provide a safe working environment. Clearly staff training and experience remain crucial in managing potentially volatile situations. Devising a more accessible reporting system so that proper levels of aggression can be easily recorded would also be beneficial. Bearing in mind that the life experiences of individual staff play a significant role in how they react to violence in the clinical area (Ferns, 2006), providing support for staff in the immediate original papers 
aftermath of an incident and in the longer term should also be considered. Future research could focus on management of patients and support of staff.

original papers

\section{Declaration of interest}

None.

\section{References}

BUCKLEY P. F., HROUDA D. R., FRIEDMAN, L., et al (2004) Insight and its relationship to violent behaviour in patients with schizophrenia. American Journal of Psychiatry, 161, 1712-1724.

CHOU, K. R., LU, R. B. \& MAO, W. C. (2002) Factors relevant to patient assaultive behavior and assault in acute in-patient psychiatric units inTaiwan. Archives of Psychiatric Nursing, 16, 187-195.

DAVID, A.S. (1990) Insight and psychosis. British Journal of Psychiatry, $156,798-808$

DEPARTMENT OF HEALTH (2002) National Minimum Standards for General Adult Services in Psychiatric Intensive Care Units (PICS) and Low Secure Environments. Department of Health Publications.

EL-BADRI, S. M. \& MELLSOP, G. (2006) Aggressive behaviour in an acute general adult psychiatric unit. Psychiatric Bulletin, 30, 166-168.

FERNS, T. (2006) Under-reporting of violent incidents against nursing staff. Nursing Standards, 40, 41-45.

FOLEY, S. R., KELLY, B. D., CLARKE, M., et al (2005) Incidence and clinical correlates of aggression and violence at presentation in patients with first episode psychosis. Schizophrenia

GRASSI, L., PERON, L., MARANGONI, C., et al (2001) Characteristics of violent behaviour in acute psychiatric inpatients: a 5-year Italian study. Acta Psychiatrica Scandinavica, 104, $273-$ 279

JAMES, D. V., FINEBERG, N. A., SHAH, A. K., et al (1990) An increase in violence on an acute psychiatric ward. A study of associated factors. British Journal of Psychiatry, 156, 846-852. Research, 72, 161-168.
NIJMAN, H. L., ALLERTZ, W.W. F., MERCKELBACH, H. L. G. J., et al (1997) Aggressive behaviour on an acute psychiatric admissions ward. European Journal of Psychiatry, 11, 106-114.

NIJMAN, H. L., MURIS, P.,

MERCKELBACH, H. L. G. J., et al (1999) The Staff Observation Aggression Scale - Revised (SOAS-R) Aggressive Behaviour, 25, 197-209.

NIJMAN, H. L., PALMSTIERNA, T ALMVIK, R., et al (2005) Fifteen years of research with the Staff Observation Aggression Scale: a review. Acta Psychiatrica Scandinavica, 111, 12-21. The Brief Psychiatric Rating Scale (BPRS). Psychological Reports, 10, 799-812.

ROSSI, A. M., JACOBS, M. MONTELEONE, M., et al (1985) Violent or fear-inducing behaviour associated with hospital admission. Hospital and Community Psychiatry, 36, 643-647.

SAVERIMUTTU, A. \& LOWE,T. (2000) Aggressive incidents on a psychiatric
OVERALL, J. E. \& GORHAM, D. R. (1962)

intensive care unit. Nursing Standards, 35, 33-36.

SHAH, A. K., FINEBERG, N. A.\& JAMES, D.V. (1991) Violence among psychiatric in-patients. Acta Psychiatrica Scandinavica, 184, 305-309.

STEINERT,T.,WIEBE, C. \& GEBHARDT, R. P. (1999) Aggressive behaviour against self and others among firstadmission patients with schizophrenia. Psychiatry Services, 50, 85-90.

SWANSON, J.W., HOLZER, C. E., GANJU, V. K., et al (1990) Violence and psychiatric disorder in the community: evidence from the epidemiologic catchment area surveys. Hospital \& Community Psychiatry, 41,761-770.

TARDIFF, K., MARZUK, P. M., LEON, A. C., et al (1997) A prospective study of violence by psychiatric patients after hospital discharge. Psychiatry Services, 48, 678-681.

WALSH, E., GILVARRY, C., SAMELE, C. et al (2004) UK700 Group. Predicting violence in schizophrenia: a prospective study. Schizophrenia Research, 67, 247-252.

Dermot P. Cohen Registrar, Mohammed Saleem Akhtar Senior Registrar, Adeel Siddiqui Registrar, Cathy Shelley Clinical Nurse Manager II, Conal Larkin Medical Director and Consultant General Adult Psychiatrist, Anthony Kinsella Statistician, St John of God Hospital, Stillorgan, Co Dublin, Eadbhard O'Callaghan Professor of Psychiatry and Consultant Adult Psychiatrist, Cluain Mhuire Family Centre, Blackrock, Co Dublin, *Abbie Lane Consultant General Adult Psychiatrist, Dublin County Stress Clinic, St John of God Hospital, Stillorgan, Co Dublin, email: abbie.lane@sjog.ie

\section{REUVEN MANFRED MAGNES}

\section{Out-patient appointments: a necessary evil? A literature review and survey of patient attendance records}

\author{
AIMS AND METHOD \\ To describe the effect of a postal \\ reminder system on UK adult \\ psychiatry clinic attendance. A litera- \\ ture review was completed and a \\ serial cross-sectional survey of \\ patient attendance records in an \\ inner-city psychiatric hospital during \\ 2006 and 2007 was undertaken.
}

\author{
RESULTS \\ A simple postal prompt reduces non- \\ attendance by up to $50 \%$ and data \\ from the serial cross-sectional survey \\ of attendance records $(n=36)$ \\ powered at $77 \%$ supported this \\ finding. Postal prompts in the survey \\ accounted for $30 \%$ improvement in \\ the variance $\left(r^{2}\right)$.
}

\author{
CLINICAL IMPLICATIONS \\ A simple postal prompt that takes \\ less than 30 s to read, sent up to \\ 2 weeks prior to the appointment \\ improves attendance by up to $50 \%$ \\ and is useful for maintaining \\ standards of excellence.
}

Missed appointments continue to be a major contributor to wasted resources in planned mental healthcare services and non-attendance is an area of concern for all healthcare providers. The national figure of $12 \%$ for nonattendance at out-patient clinics in the UK hides large variations between specialties and between regions. Studies report figures that range from 5\% to 34\% (Sharp \& Hamilton, 2001). A Cochrane review (Reda \& Makhoul, 2001) suggests that a very simple prompt to attend clinic is financially sound advice for managers as well as being good clinical practice.

\section{Method}

\section{Literature search}

A literature review of peer-reviewed psychiatry journals 1974-2007 was conducted. A number of databases were searched (EMBASE, MEDLINE, PsycLit, King's Fund, CINAHL, PsycINFO) with keywords: 'psychiatry outpatient', 'improve out-patient attendance', 'nonattendance'. The search was limited to papers published in English and peer-reviewed journals. Among the publications found, seven were suitable for inclusion (Rusius, 